\title{
Strong Couplings of Charmed Mesons and Quarkonia
}

\section{Wolfgang Lucha}

Institute for High Energy Physics, Austrian Academy of Sciences, Nikolsdorfergasse 18, A-1050

Vienna, Austria

E-mail: Wolfgang. Lucha@oeaw.ac.at

\section{Dmitri Melikhov*}

D. V. Skobeltsyn Institute of Nuclear Physics, Moscow State University, 119991, Moscow,

Russia, and

Faculty of Physics, University of Vienna, Boltzmanngasse 5, A-1090 Vienna, Austria

E-mail: dmitri_melikhov@gmx.de

\section{Hagop Sazdjian}

IPN, CNRS/IN2P3, Universitè Paris-Sud 11, F-91406 Orsay, France

E-mail: sazdjian@ipno.in2p3.fr

\section{Silvano Simula}

INFN, Sezione di Roma Tre, Via della Vasca Navale 84, I-00146 Roma, Italy

E-mail: simula@roma3.infn.it

We extract the strong coupling constants of three mesons, each of which is composed of either two charm quarks or one charm quark and one light (i.e., $u, d$, or $s$ ) quark, from the matrix elements for the transitions of two of these mesons induced by appropriate quark currents within the framework of a relativistic dispersion approach to the constituent-quark picture of mesons. Among others, we also analyse the impact of the violation of the SU(3) flavour symmetry by the quark masses. In the case of mesons containing one light quark, we observe, in two respects, discrepancies between our findings and the predictions of QCD sum rules: our strong couplings exceed considerably the ones emerging from QCD sum rules, and, in our approach, the replacement of a light quark by a strange quark entails, in contrast to QCD sum rules, a reduction of the magnitudes of the strong couplings.

The European Physical Society Conference on High Energy Physics

22-29 July 2015

Vienna, Austria

\footnotetext{
* Speaker.
} 


\section{Lots of definitions: strong couplings, transition form factors, and decay constants}

By use of a relativistic dispersion approach relying on the constituent-quark model for hadrons, we analyse the three-meson strong couplings $g_{P P^{\prime} V}$ and $g_{P V^{\prime} V}$ for vector mesons $V$, with polarization vectors $\varepsilon_{\mu}$, and pseudoscalar mesons $P$ : physical parameters defined by the three-meson amplitudes

$$
\begin{aligned}
\left\langle P^{\prime}\left(p_{2}\right) V(q) \mid P\left(p_{1}\right)\right\rangle & =-\frac{g_{P P^{\prime} V}}{2}\left(p_{1}+p_{2}\right)^{\mu} \varepsilon_{\mu}^{*}(q), \\
\left\langle V^{\prime}\left(p_{2}\right) V(q) \mid P\left(p_{1}\right)\right\rangle & =-g_{P V^{\prime} V} \varepsilon_{\mathcal{E}^{*}}(q) \varepsilon^{*}\left(p_{2}\right) p_{1} p_{2}, \quad q \equiv p_{1}-p_{2} .
\end{aligned}
$$

Of particular interest to us will be any system with, at least, one of the charmonia, $J / \psi$ or $\eta_{c}$, among the meson triple. These strong couplings enter into the residues of poles in corresponding transition form factors for $q^{2}>0$ arising from intermediate meson states. The form factors relevant for us read

$$
\begin{aligned}
\left\langle P^{\prime}\left(p_{2}\right)\left|\bar{q}_{2} \gamma_{\mu} q_{1}\right| P\left(p_{1}\right)\right\rangle & =F_{+}^{P \rightarrow P^{\prime}}\left(q^{2}\right)\left(p_{1}+p_{2}\right)_{\mu}+\cdots, \\
\left\langle V\left(p_{2}\right)\left|\bar{q}_{2} \gamma_{\mu} q_{1}\right| P\left(p_{1}\right)\right\rangle & =\frac{2 V^{P \rightarrow V}\left(q^{2}\right)}{M_{P}+M_{V}} \varepsilon_{\mu \varepsilon^{*}\left(p_{2}\right) p_{1} p_{2},} \\
\left\langle V\left(p_{2}\right)\left|\bar{q}_{2} \gamma_{\mu} \gamma_{5} q_{1}\right| P\left(p_{1}\right)\right\rangle & =\mathrm{i} q_{\mu}\left(\varepsilon^{*}\left(p_{2}\right) p_{1}\right) \frac{2 M_{V}}{q^{2}} A_{0}^{P \rightarrow V}\left(q^{2}\right)+\cdots .
\end{aligned}
$$

The decay constants $f_{V}$ and $f_{P}$ of vector and pseudoscalar mesons are defined, by meson-to-vacuum transition amplitudes of vector quark currents $\bar{q}_{1} \gamma_{\mu} q_{2}$ and axial-vector quark currents $\bar{q}_{1} \gamma_{\mu} \gamma_{5} q_{2}$, by

$$
\left\langle 0\left|\bar{q}_{1} \gamma_{\mu} q_{2}\right| V(q)\right\rangle=f_{V} M_{V} \varepsilon_{\mu}(q), \quad\left\langle 0\left|\bar{q}_{1} \gamma_{\mu} \gamma_{5} q_{2}\right| P(q)\right\rangle=\mathrm{i} f_{P} q_{\mu} .
$$

The poles of the form factors, residing at pseudoscalar or vector resonances $P_{R}$ or $V_{R}$, are of the form

$$
\begin{aligned}
F_{+}^{P \rightarrow P^{\prime}}\left(q^{2}\right) & =\frac{g_{P P^{\prime} V_{R}} f_{V_{R}}}{2 M_{V_{R}}} \frac{1}{1-\frac{q^{2}}{M_{V_{R}}^{2}}}+\cdots, \\
V^{P \rightarrow V}\left(q^{2}\right) & =\frac{\left(M_{V}+M_{P}\right) g_{P V V_{R}} f_{V_{R}}}{2 M_{V_{R}}} \frac{1}{1-\frac{q^{2}}{M_{V_{R}}^{2}}}+\cdots, \\
A_{0}^{P \rightarrow V}\left(q^{2}\right) & =\frac{g_{P P_{R} V} f_{P_{R}}}{2 M_{V}} \frac{1}{1-\frac{q^{2}}{M_{P_{R}}^{2}}}+\cdots
\end{aligned}
$$

\section{Quark-model-based dispersion approach}

We calculate the three relevant form factors, viz., $F_{+}^{P \rightarrow P^{\prime}}\left(q^{2}\right), V^{P \rightarrow V}\left(q^{2}\right)$, and $A_{0}^{P \rightarrow V}\left(q^{2}\right)$, within the framework of a relativistic constituent-quark picture [1]. To this end, we must relate the currents defining the form factors to their constituent-quark $(Q)$ counterparts. The task of establishing such a relationship is easily accomplished for heavy-quark currents by introducing form factors $g_{V}$ and $g_{A}$,

$$
\bar{q}_{1} \gamma_{\mu} q_{2}=g_{V} \bar{Q}_{1} \gamma_{\mu} Q_{2}+\cdots, \quad \bar{q}_{1} \gamma_{\mu} \gamma_{5} q_{2}=g_{A} \bar{Q}_{1} \gamma_{\mu} \gamma_{5} Q_{2}+\cdots,
$$

but is not that easy for the case of light quarks [2]. Numerically, we use [3] for the constituent-quark masses and couplings $m_{d}=m_{u}=0.23 \mathrm{GeV}, m_{s}=0.35 \mathrm{GeV}, m_{c}=1.45 \mathrm{GeV}$, and $g_{V}=g_{A}=1$. The relativistic dispersion technique enables us to represent all the quantities of interest by integrals over 
invariant masses of intermediate quark-antiquark states of spectral densities derived from one-loop Feynman diagrams and wave functions of the relevant pseudoscalar or vector mesons [4] of the form

$$
\begin{aligned}
& \phi_{P, V}(s)=\frac{\pi}{\sqrt{2}} \frac{\sqrt{s^{2}-\left(m_{1}^{2}-m^{2}\right)^{2}}}{\sqrt{s-\left(m_{1}-m\right)^{2}}} \frac{w_{P, V}\left(k^{2}\right)}{s^{3 / 4}}, \\
& k^{2}=\frac{\left(s-m_{1}^{2}-m^{2}\right)^{2}-4 m_{1}^{2} m^{2}}{4 s}, \quad \int \mathrm{d} k k^{2} w_{P, V}^{2}\left(k^{2}\right)=1 .
\end{aligned}
$$

For the radial meson wave functions $w_{P, V}\left(k^{2}\right)$, we'll find sufficient to assume some Gaussian shapes

$$
w_{P, V}\left(k^{2}\right) \propto \exp \left(-\frac{k^{2}}{2 \beta_{P, V}^{2}}\right)
$$

fixed by parameters $\beta_{P, V}$. Within the dispersion formalism, the decay constants $f_{P, V}$ become spectral integrals of densities $\rho_{P, V}(s)$, and the form factors $F_{+}^{P \rightarrow P^{\prime}}\left(q^{2}\right), V^{P \rightarrow V}\left(q^{2}\right)$ and $A_{0}^{P \rightarrow V}\left(q^{2}\right)$, generically called $\mathscr{F}\left(q^{2}\right)$, become double dispersion integrals of suitable double spectral densities $\Delta\left(s_{1}, s_{2}, q^{2}\right)$ :

$$
f_{P, V}=\int \mathrm{d} s \phi_{P, V}(s) \rho_{P, V}(s), \quad \mathscr{F}\left(q^{2}\right)=\int \mathrm{d} s_{1} \phi_{1}\left(s_{1}\right) \int \mathrm{d} s_{2} \phi_{2}\left(s_{2}\right) \Delta\left(s_{1}, s_{2}, q^{2}\right) .
$$

The double spectral densities $\Delta\left(s_{1}, s_{2}, q^{2}\right)$ can be rather straightforwardly derived from the Feynman diagrams shown in Fig. 1. Table 1 subsumes the required parameter values of the mesons discussed.

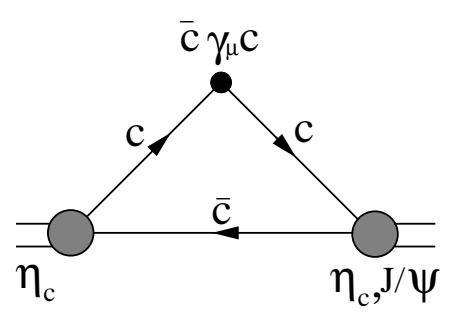

(a)

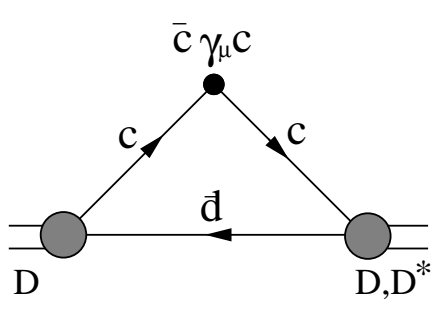

(b)

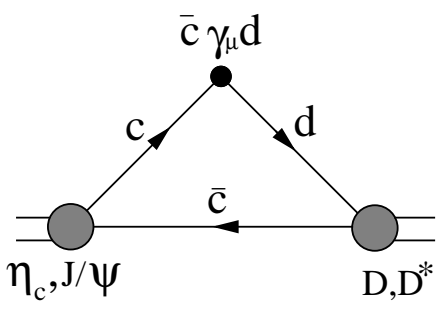

(c)

Figure 1: (One-loop) Feynman graphs for transitions induced by constituent-quark vector currents $\bar{Q}_{1} \gamma_{\mu} Q_{2}$.

Table 1: Masses $M$, decay constants $f_{P, V}$ and slope parameters $\beta_{P, V}$ of the $D_{(s)}^{(*)}$ mesons and charmonia $c \bar{c}$ [5].

\begin{tabular}{lcccccc}
\hline \hline \multicolumn{1}{c}{ Meson } & $D$ & $D^{*}$ & $D_{s}$ & $D_{s}^{*}$ & $\eta_{c}$ & $J / \psi$ \\
\hline$M(\mathrm{GeV})$ & 1.87 & 2.010 & 1.97 & 2.11 & 2.980 & 3.097 \\
$f_{P, V}(\mathrm{MeV})$ & $206 \pm 8$ & $260 \pm 10$ & $248 \pm 2.5$ & $311 \pm 9$ & $394.7 \pm 2.4$ & $405 \pm 7$ \\
$\beta_{P, V}(\mathrm{GeV})$ & 0.475 & 0.48 & 0.545 & 0.54 & 0.77 & 0.68 \\
\hline \hline
\end{tabular}

All form factors $\mathscr{F}$, computed off the actual locations of their resonances $R$, may be interpolated by

$$
\mathscr{F}\left(q^{2}\right)=\frac{\mathscr{F}(0)}{\left(1-\frac{q^{2}}{M_{R}^{2}}\right)\left(1-\frac{\sigma_{1} q^{2}}{M_{R}^{2}}+\frac{\sigma_{2} q^{4}}{M_{R}^{4}}\right)}, \quad \operatorname{Res} \mathscr{F}\left(M_{R}^{2}\right)=\frac{\mathscr{F}(0)}{1-\sigma_{1}+\sigma_{2}} .
$$

From the residues of these form factors - involving the meson masses, decay constants, and strong couplings - the latter are derived, by combined fits if they are present in more than one form factor. 


\section{Strong couplings of three charmonia: $\eta_{c} \eta_{c} J / \psi$ and $\eta_{c} J / \psi J / \psi$}

As one example representative of the general situation, we inspect the strong couplings of three charmonia [6]. More precisely, we start with the strong coupling $g_{\eta_{c}} \eta_{c} \psi$ of one vector $J / \psi$ meson to two pseudoscalar $\eta_{c}$ mesons; the latter strong coupling enters in and therefore can be extracted from the residue $\operatorname{Res} F_{+}^{\eta_{c} \rightarrow \eta_{c}}\left(M_{\psi}^{2}\right)$ of the pole at $q^{2}=M_{\psi}^{2}$ of the form factor $F_{+}^{\eta_{c} \rightarrow \eta_{c}}\left(q^{2}\right)$ for the ("elastic") transition $\eta_{c} \rightarrow \eta_{c}$ provoked by the vector quark current $\bar{c} \gamma_{\mu} c$ which couples with decay constant $f_{\psi}$ to the vector-meson resonance $J / \psi$ and from the residue $\operatorname{Res} A_{0}^{\eta_{c} \rightarrow \psi}\left(M_{\eta_{c}}^{2}\right)$ of the pole at $q^{2}=M_{\eta_{c}}^{2}$ of the form factor $A_{0}^{\eta_{c} \rightarrow \psi}\left(q^{2}\right)$ for the transition $\eta_{c} \rightarrow J / \psi$ effectuated by the axial-vector quark current $\bar{c} \gamma_{\mu} \gamma_{5} c$ which couples with decay constant $f_{\eta_{c}}$ to the pseudoscalar-meson resonance $\eta_{c}$. These read

$$
\begin{aligned}
& \operatorname{Res} F_{+}^{\eta_{c} \rightarrow \eta_{c}}\left(M_{\psi}^{2}\right)=g \eta_{c} \eta_{c} \psi \frac{f_{\psi}}{2 M_{\psi}}, \\
& \operatorname{Res} A_{0}^{\eta_{c} \rightarrow \psi}\left(M_{\eta_{c}}^{2}\right)=g \eta_{\eta_{c}} \eta_{c} \psi \frac{f_{\eta_{c}}}{2 M_{\psi}} .
\end{aligned}
$$

Upon determination of all needed meson wave-function parameters $\beta_{P, V}$ by requiring the dispersion representation of the decay constants $f_{P, V}$ to reproduce their (observed) values, the strong couplings may be calculated, individually for each transition of interest, from the spectral representation of the relevant form factors: our couplings' off-resonance behaviour exhibits excellent agreement (Fig. 2).

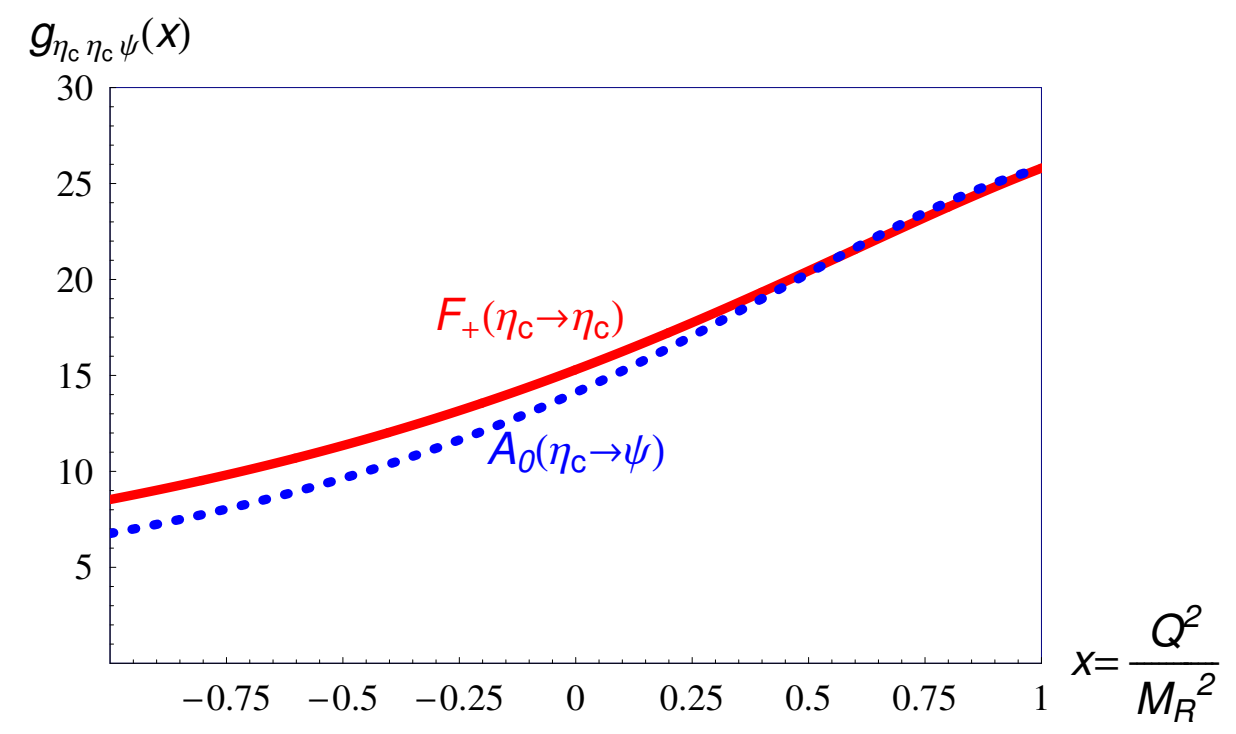

Figure 2: Dependence on $x \equiv \frac{q^{2}}{M_{R}^{2}}$ of the "off-shell strong coupling" $g_{\eta_{c}} \eta_{c} \psi(x): g_{\eta_{c}} \eta_{c} \widehat{\psi}(x)$, from the transition $\eta_{c} \rightarrow \eta_{c}$ (resonance $R=J / \psi$, red), versus $g_{\eta_{c} \widehat{\eta}_{c} \psi}(x)$, from the transition $\eta_{c} \rightarrow J / \psi$ (resonance $R=\eta_{c}$, blue).

Recalling $F_{+}^{\eta_{c} \rightarrow \eta_{c}}(0)=1$, a combined fit with the four parameters $g_{\eta_{c} \eta_{c} \psi}, A_{0}^{\eta_{c} \rightarrow \psi}(0), \sigma_{1}^{F, A}$ yields [6]

$$
g_{\eta_{c} \eta_{c} \psi}=25.8 \pm 1.7 \text {. }
$$

Proceeding along a similar path, we predict [6] for the strong coupling $g_{\eta_{c} \psi \psi}$ of one pseudoscalar $\eta_{c}$ meson to two vector $J / \psi$ mesons (which turns out to enter in only a single meson-meson transition)

$$
g_{\eta_{c} \psi \psi}=(10.6 \pm 1.5) \mathrm{GeV}^{-1} \text {. }
$$




\section{Strong couplings of the charmonia $\eta_{c}$ or $J / \psi$ to the charmed mesons $D_{(s)}$ and $D_{(s)}^{*}$}

Allowing also for participation of quark currents with a $d$ or $s$ quark in the game and "merging" strong-coupling multiple occurrences (all of them showing nearly perfect concord, Fig. 3) yields [6]

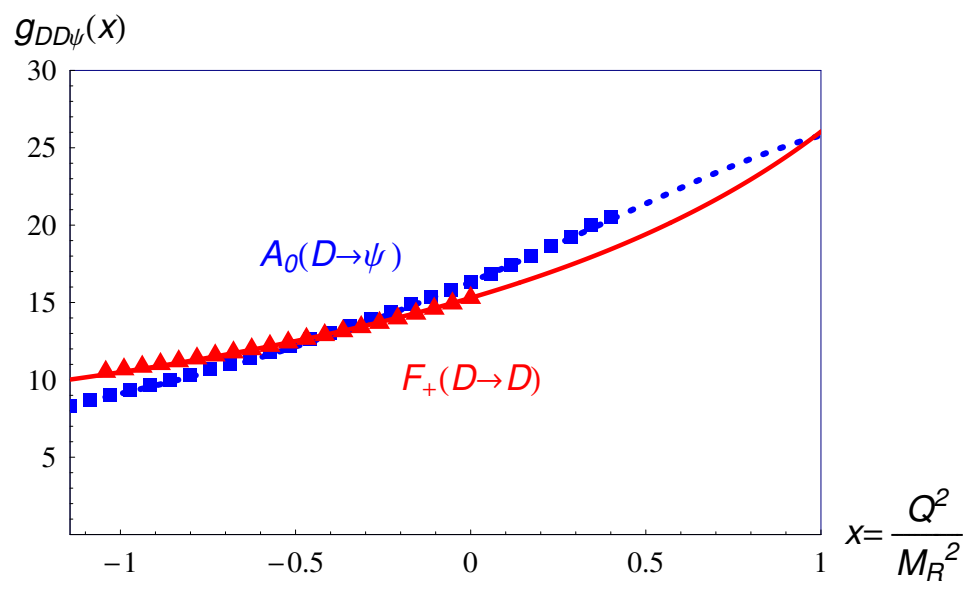

(a)

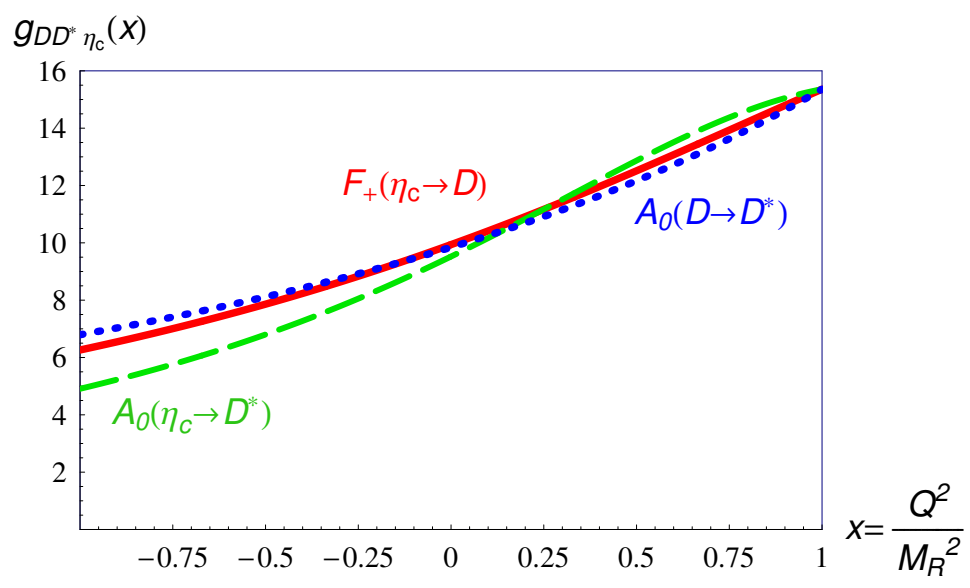

(b)

Figure 3: Dependence on $x \equiv \frac{q^{2}}{M_{R}^{2}}$ of "off-shell strong couplings": (a) $g_{D D \widehat{\psi}}(x)=\frac{2 M_{\psi}}{f_{\psi}}(1-x) F_{+}^{D \rightarrow D}\left(q^{2}\right)$ (red) or $g_{D \widehat{D} \psi}(x)=\frac{2 M_{\psi}}{f_{D}}(1-x) A_{0}^{D \rightarrow \psi}\left(q^{2}\right)$ (blue), and, similarly, (b) $g_{D \widehat{D}^{*} \eta_{c}}(x)$ (red), $g_{D D^{*} \widehat{\eta}_{c}}(x)$ (blue) or $g_{\widehat{D} D^{*} \eta_{c}}(x)$ (green). For clarity, a circumflex over the particle's symbol serves to identify the respective resonance meson.

- for the strong couplings of the charmonia $J / \psi$ or $\eta_{c}$ to the non-strange charmed mesons $D^{(*)}$,

$$
\begin{array}{ll}
g_{D D \psi}=26.04 \pm 1.43, & g_{D D^{*} \psi}=(10.7 \pm 0.4) \mathrm{GeV}^{-1}, \\
g_{D D^{*} \eta_{c}}=15.51 \pm 0.45, & g_{D^{*} D^{*} \eta_{c}}=(9.76 \pm 0.32) \mathrm{GeV}^{-1},
\end{array}
$$

- and, for the strong couplings of the charmonia $J / \psi$ or $\eta_{c}$ to the strange charmed mesons $D_{s}^{(*)}$,

$$
\begin{array}{ll}
g_{D_{s} D_{s} \psi}=23.83 \pm 0.78, & g_{D_{s} D_{s}^{*} \psi}=(9.6 \pm 0.8) \mathrm{GeV}^{-1}, \\
g_{D_{s} D_{s}^{*} \eta_{c}}=14.15 \pm 0.52, & g_{D_{s}^{*} D_{s}^{*} \eta_{c}}=(8.27 \pm 0.37) \mathrm{GeV}^{-1} .
\end{array}
$$




\section{Observations, comparison, and conclusions}

Our present study of the interaction strengths parametrizing the strong couplings of all possible three-particle combinations of charmonia and charmed mesons produced a variety of unexpected, if not even astounding observations: First, the successful extrapolation of our interpolated findings for strong couplings obtained at $q^{2}<0$ confirms the existence of the poles expected for $q^{2}>0$. Second, concerning SU(3) breaking, the net result of replacing the $d$ quark by an $s$ quark is a reduction of the affected strong coupling by some $10 \%$. Third, despite undeniable similarities of the approaches, our $D_{(s)}^{(*)}$ couplings [6] are more than twice as large as those emerging from QCD sum rules (cf. Table 2).

Table 2: Confrontation of our strong-coupling predictions with corresponding QCD sum-rule figures [7 -9].

\begin{tabular}{lcccc}
\hline \hline Strong coupling & $g_{D D \psi}$ & $g_{D D^{*} \psi}\left(\mathrm{GeV}^{-1}\right)$ & $g_{D_{s} D_{s} \psi}$ & $g_{D_{s} D_{s}^{*} \psi}\left(\mathrm{GeV}^{-1}\right)$ \\
\hline This investigation [6] & $26.04 \pm 1.43$ & $10.7 \pm 0.4$ & $23.83 \pm 0.78$ & $9.6 \pm 0.8$ \\
QCD sum rules & $11.6 \pm 1.8[7]$ & $4.0 \pm 0.6[7]$ & $11.96 \pm 1.34[8]$ & $4.30 \pm 1.53[9]$ \\
\hline \hline
\end{tabular}

\section{References}

[1] W. Lucha, F. F. Schöberl, and D. Gromes, Phys. Rep. 200 (1991) 127; F. Cardarelli, E. Pace, G. Salmè, and S. Simula, Phys. Lett. B 357 (1995) 267, arXiv:nucl-th/9507037; R. N. Faustov and V. O. Galkin, Z. Phys. C 66 (1995) 119; V. Anisovich, D. Melikhov, and V. Nikonov, Phys. Rev. D 52 (1995) 5259, arXiv:hep-ph/9503473; V. V. Anisovich, D. I. Melikhov, and V. A. Nikonov, Phys. Rev. D 55 (1997) 2918, arXiv:hep-ph/9607215.

[2] D. Melikhov and B. Stech, Phys. Rev. D 74 (2006) 034022, arXiv:hep-ph/0606203; W. Lucha, D. Melikhov, and S. Simula, Phys. Rev. D 74 (2006) 054004, arXiv:hep-ph/0606281.

[3] D. Melikhov and B. Stech, Phys. Rev. D 62 (2000) 014006, arXiv:hep-ph/0001113.

[4] D. Melikhov, Eur. Phys. J. direct C4 (2002) 2, arXiv:hep-ph/0110087.

[5] C. T. H. Davies et al., Phys. Rev. D 82 (2010) 114504, arXiv:1008.4018 [hep-lat]; W. Lucha, D. Melikhov, and S. Simula, Phys. Lett. B 701 (2011) 82, arXiv:1101.5986 [hep-ph]; D. Bečirević et al., JHEP 1202 (2012) 042, arXiv:1201.4039 [hep-lat]; G. C. Donald et al., Phys. Rev. D 86 (2012) 094501, arXiv:1208.2855 [hep-lat]; W. Lucha, D. Melikhov, and S. Simula, Phys. Lett. B 735 (2014) 12, arXiv:1404.0293 [hep-ph]; K. A. Olive et al. (Particle Data Group), Chin. Phys. C 38 (2014) 090001.

[6] W. Lucha, D. Melikhov, H. Sazdjian, and S. Simula, arXiv:1506.09213 [hep-ph].

[7] R. D. Matheus et al., Int. J. Mod. Phys. E 14 (2005) 555.

[8] B. Osório Rodrigues, M. E. Bracco, and M. Chiapparini, Nucl. Phys. A 929 (2014) 143; arXiv:1309.1637 [hep-ph].

[9] B. Osório Rodrigues et al., Eur. Phys. J. A 51 (2015) 28; arXiv:1501.03088 [hep-ph]. 